\title{
Male circumcision as a preventive measure limiting HIV transmission Thomas C Quinn*
}

\begin{abstract}
Address: National Institute of Allergy and Infectious Diseases, National Institutes of Health, Bethesda, Maryland, 20892, USA
\end{abstract}
* Corresponding author

from 2006 International Meeting of The Institute of Human Virology

Baltimore, USA. 17-21 November, 2006

Published: 2I December 2006

Retrovirology 2006, 3(SuppI I):SI09 doi:I0.I I86/I742-4690-3-SI-SI09

(C) 2006 Quinn; licensee BioMed Central Ltd.

From population based studies and couple studies, the probability of HIV transmission and acquisition has been associated with the blood and genital viral load, male circumcision, STDs, stage of disease, genetic haplotype, viral subtype, and levels of mucosal immune response. This presentation will review the relationship between male circumcision and HIV transmission and discuss policy implications in promoting circumcision as a prevention modality. A systematic meta-analysis of 38 studies, mostly in Africa, found that circumcised men appear to have a $50 \%$ or more reduction in HIV infection as uncircumcised men. A sub-analysis of 16 of these studies found a $70 \%$ reduction in HIV infection among higher-risk men. Mapping of the HIV epidemic has demonstrated a strong correlation between regions with higher rates of HIV infection and those with lower circumcision rates. A twoyear cohort study of male partners of HIV-positive women in Rakai, Uganda, found that 40 of 137 uncircumcised men became infected compared to 0 of 50 circumcised men over 2.1 years $(\mathrm{p}=0.0004)$. Biological explanations include greater ability of the internal foreskin to absorb HIV more efficiently due to the greater presence of Langerhans and other HIV target cells and its greater susceptibility to tears, abrasions, and consequently infection by STDs and HIV. One RCT in South Africa demonstrated that circumcision afforded a $61 \%$ (95\% CI: 34\%-77\%) reduction in HIV incidence even after controlling for behavioral factors (Auvert et al., PLoS Medicine 2005;298). Two other RCT trials of male circumcision in Uganda and Kenya are in progress. In summary, these epidemiologic, biological, and clinical trial results provide very strong evidence that male circumcision significantly lowers the risk of HIV acquisition. Mathematical models of implementing male circumcision in countries with high incident rates suggest marked reductions in HIV incidence in men with subsequent decreased transmission rates to women. Policy implications of recommending male circumcision to populations in high-risk countries need to take into consideration cultural norms, religious traditions, national and local laws. Circumcision may represent one important biological intervention to decreasing the acquisition of HIV, but will need to be carefully integrated into other HIV prevention and STD control programs in order to prevent subsequent behavioral disinhibition" among circumcised men. 